\title{
Connected Domination Numbers of Fuzzy Square Graphs
}

\author{
N.Sarala ${ }^{1}$, T.Kavitha ${ }^{2}$ \\ ${ }^{1}$ (Department of Mathematics, ADM College, Nagapattinam, Tamilnadu , India) \\ ${ }_{2}^{2}$ (Department of Mathematics, EGSP Engineering College, Nagapattinam, Tamilnadu, India
}

\begin{abstract}
The fuzzy square graph $G$ is $G^{2}=\left(V, E\left(G^{2}\right)\right)$. A set $D$ of $G^{2}$ is said to be connected dominating set of $G^{2}$ if every vertex not in $D$ is adjacent to at least one vertex in $D$ and the sub graph $H=\langle D\rangle$ is connected . The minimum cardinality of a connected dominating set of $G^{2}$ is called the connected domination number of fuzzy square graph $G^{2}$ and denoted by $\gamma_{c}\left(G^{2}\right)$. In this paper, we introduce connected dominating numbers of fuzzy square graph and proves some important results related to connected dominating numbers of fuzzy square graphs.
\end{abstract}

Keywords: Connected dominating set, dominating set, Fuzzy graphs, fuzzy square graphs.

\section{Introduction}

In 1975, the notion of fuzzy graph and several fuzzy analogues of graph theoretical concepts such as paths cycles and connectedness are introduced by Rosenfeld[4]. Bhattacharya[1] has established some connectivity regarding fuzzy cut node and fuzzy bridges. The concept of domination in fuzzy graphs are introduced by A.Somasudaram and S.Somasundaram[6] in 1998. In 2012, Bounds on connected domination in square graph of graph is introduced by M.H.Muddabihal and G.Srinivasa[7]. In this paper ,We analyze bounds on connected dominating set of fuzzy square graph and proves some results based on connected dominating fuzzy graph.

\section{Preliminaries}

Definition 2.1 [2] A fuzzy subset of a nonempty set $\mathrm{V}$ is a mapping $\sigma: \mathrm{V} \rightarrow[0,1]$ and A fuzzy relation on $\mathrm{V}$ is a fuzzy subset of $\mathrm{V} x \mathrm{~V}$. A fuzzy graph is a pair $\mathrm{G}:(\sigma, \mu)$ where $\sigma$ is a fuzzy subset of a set $\mathrm{V}$ and $\mu$ is a fuzzy relation on $\sigma$, where $\mu(\mathrm{u}, \mathrm{v}) \leq \sigma(\mathrm{x}) \Lambda \sigma(\mathrm{y}) \forall \mathrm{x}, \mathrm{y} \in \mathrm{V}$

Definition 2.2[2] The fuzzy graph $\mathrm{H}\left(\mathrm{V}_{1}, \sigma^{\prime}, \mu^{\prime}\right)$ is called a fuzzy subgraph of $\mathrm{G}(\mathrm{V}, \sigma, \mu)$ if $\mathrm{V}_{1} \subseteq \mathrm{V}, \sigma^{\prime}(\mathrm{u}) \leq \sigma(\mathrm{u})$ for all $\mathrm{u} \in \mathrm{V}$ and $\mu^{\prime}(\mathrm{u}, \mathrm{v}) \leq \mu(\mathrm{u}, \mathrm{v})$ for all $\mathrm{u}, \mathrm{v} \in \mathrm{V}$

Definition 2.3 [2] The fuzzy subgraph $\mathrm{H}\left(\mathrm{V}_{1}, \sigma^{\prime}, \mu^{\prime}\right)$ is said to be a spanning fuzzy subgraph of $\mathrm{G}(\mathrm{V}, \sigma, \mu)$ if $\sigma(\mathrm{u})=\sigma^{\prime}(\mathrm{u})$ for all $\mathrm{u} \in \mathrm{V}_{1}$ and $\mu^{\prime}(\mathrm{u}, \mathrm{v}) \leq \mu(\mathrm{u}, \mathrm{v})$ for all $\mathrm{u}, \mathrm{v} \in \mathrm{V}$.

Definition 2.4 [2] The fuzzy graph $H(\tau, \rho)$ is called a partial fuzzy subgraph of $G(V, \sigma, \mu)$ if $\tau \leq \sigma$ and $\rho \leq \mu$.

The partial fuzzy subgraph of $\mathrm{G}(\mathrm{V}, \sigma, \mu)$ induced by $\tau$ is maximal partial fuzzy subgraph of $\mathrm{G}$ that has fuzzy vertex set $\tau$. This is partial fuzzy graph $\mathrm{H}(\tau, \rho)$ where $\rho(\mathrm{x}, \mathrm{y})=\tau(\mathrm{x}) \Lambda \tau(\mathrm{y}) \Lambda \mu(\mathrm{x}, \mathrm{y}) \forall \mathrm{x}, \mathrm{y} \in \mathrm{V}$.

Definition 2.5[9] The Strength of the connectedness between two nodes $\mathrm{u}, \mathrm{v}$ in a fuzzy graph $\mathrm{G}$ is $\mu^{\infty}(\mathrm{u}, \mathrm{v})=\sup \left\{\mu^{\mathrm{k}}(\mathrm{u}, \mathrm{v}) ; \mathrm{k}=1,2,3 ..\right\} \quad$ where $\mu^{\mathrm{k}}(\mathrm{u}, \mathrm{v})=\sup \mu\left(\mathrm{u}, \mathrm{u}_{1}\right) \Lambda \mu\left(\mathrm{u}_{1}, \mathrm{u}_{2}\right) \Lambda \mu\left(\mathrm{u}_{2}, \mathrm{u}_{3}\right) \Lambda \ldots . \mu\left(\mathrm{u}_{\mathrm{k}-1, \mathrm{~V}}\right)$. An $\operatorname{arc}(\mathrm{u}, \mathrm{v})$ is said to be a strong arc if $\mu(\mathrm{u}, \mathrm{v})=\mu^{\infty}(\mathrm{u}, \mathrm{v})$. If $\mu(\mathrm{u}, \mathrm{v})=0$ for every $\mathrm{v} \in \mathrm{V}$ then $\mathrm{u}$ is called isolated node.

Definition 2.6[2] An arc (u,v) is called a fuzzy bridge in $G$, if the removal of $(u, v)$ reduces the strength of connectedness between some pair of nodes in G.A connected fuzzy graph is called a fuzzy tree, if it contains a spanning subgraphs $\mathrm{T}$ which is a tree such that for all $\operatorname{arcs}(\mathrm{u}, \mathrm{v})$ not in $\mathrm{T}$.

Definition 2.7 [9] Let $\mathrm{G}(\mathrm{V}, \sigma, \mu)$ be a fuzzy graph and $\mathrm{D} \subseteq \mathrm{V}$. D is a dominating set if for every $\mathrm{u} \in \mathrm{V}$-D there exist $\mathrm{v} \in \mathrm{D}$ such that $(\mathrm{u}, \mathrm{v})$ is strong arc and $\sigma(\mathrm{u}) \leq \sigma(\mathrm{v})$. 


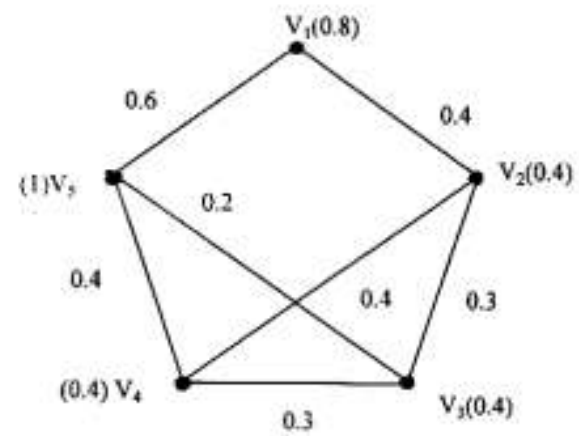

Fig.1. $\mathrm{D}=\left\{\mathrm{V}_{3}, \mathrm{~V}_{3}\right\}$

Definition 2.8 [9] A dominating set of a fuzzy graph with minimum number of vertices is called a minimum dominating set. The domination number of $\mathrm{G}$ is denoted by $\gamma(\mathrm{G})$. Domination number of a fuzzy graph is the sum of membership values of the vertices of a minimum dominating set.

\section{Connected Dominating Set In Fuzzy Graphs}

Definition 3.1 [10] . A dominating set D of a fuzzy graph $G=(\sigma, \mu)$ is connected dominating set if the induced fuzzy subgraph $\mathrm{H}=\langle\mathrm{D}\rangle$ is connected. The minimum cardinality of a connected dominating set of $\mathrm{G}$ is called the connected dominating number of $G$ and is denoted by $\gamma_{c}(G)$, obviously $\gamma(G) \leq \gamma_{c}(G)$.

In this section, We proved some important theorems based on connected dominating fuzzy graphs.

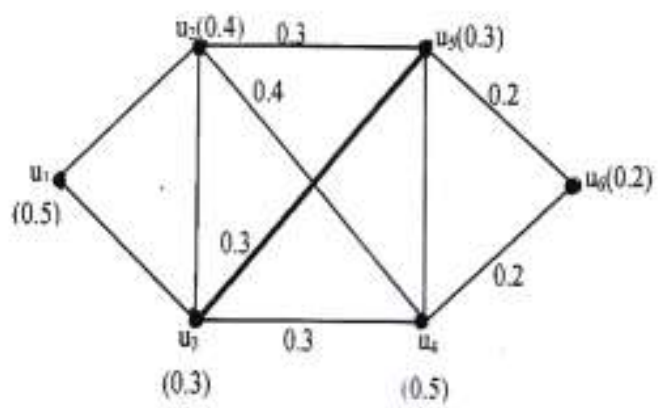

Fig. 2. $D=\left\{u_{3}, u_{s}\right\}, \gamma_{t}(G)=0.6$

Theorem 3.2 : Let $\mathrm{G}=(\mathrm{V}, \sigma, \mu)$ be a connected fuzzy graph order $\mathrm{n}$ and let $\gamma_{\mathrm{c}}(\mathrm{G})=\mathrm{d}$, let $\mathrm{S} \subseteq \mathrm{V}$ be such that $\langle\mathrm{S}\rangle$ is connected then $|\mathrm{N}(\mathrm{S})-\mathrm{S}| \leq \mathrm{n}-\mathrm{d}$.

Proof : The result is clear if $\mathrm{d} \leq|\mathrm{S}|$ so assume $\mathrm{d}>|\mathrm{S}|$. If $\mathrm{V}-\mathrm{S}-\mathrm{N}(\mathrm{S})=\Phi$ then $\gamma_{\mathrm{c}}(\mathrm{G})<\mathrm{d}$ a contradiction.

Let $\mathrm{H}=\left\langle\mathrm{V}-\mathrm{S}-\mathrm{N}(\mathrm{S})>\right.$ and let $\mathrm{H}_{1}, \mathrm{H}_{2}, . . \mathrm{H}_{l}$ be the connected components of $\mathrm{H}$. Because $\mathrm{G}$ is connected for each $\mathrm{i}, l \leq \mathrm{i} \leq l$ there exists $\mathrm{u}_{\mathrm{i}} \in \mathrm{N}(\mathrm{S})$ and $\mathrm{v}_{\mathrm{i}} \in \mathrm{V}\left(\mathrm{H}_{\mathrm{i}}\right)$ Such that $\mathrm{u}_{\mathrm{i}}$ is adjacent to $\mathrm{v}_{\mathrm{i}}$ where $\mu\left(\mathrm{u}_{\mathrm{i}}, \mathrm{v}_{\mathrm{i}}\right)>0 \quad \forall \mathrm{i}$. The subgraph induced by $X$ is connected and that $X$ dominates G. Hence $d \leq|x| \leq|v|-|N(S)-S|$ that is $|N(S)-S| \leq|v|$ $-d$

Corollary 3.3 : If $G$ is connected and has $n$ vertices and $\gamma_{c}(G)=d$ then every vertex in $G$ has degree atmost n-d.

Proof : Apply the proceeding theorem with S consisting of just one vertex.

Theorem 3.4: If $G$ is connected fuzzy graph and $n \geq 3$ then $\gamma_{c}(G) \leq n-2$

Proof: Let $\mathrm{T}\left(\mathrm{V}_{1}, \tau, \rho\right)$ be a spanning fuzzy tree of $\mathrm{G}$ such that $\tau(\mathrm{u})=\sigma(\mathrm{u})$ for all $\mathrm{u} \in \mathrm{V}_{1}$ and $\rho(\mathrm{u}, \mathrm{v}) \leq$ $\mu(\mathrm{u}, \mathrm{v})$ with $\varepsilon_{\mathrm{T}}(\mathrm{G})$ pendant vertices and Let $\mathrm{L}$ denote the set of pendant vertices then T-L is connected dominating set having $n-\varepsilon_{T}(G)$ vertices. that is $\gamma_{c}(G) \leq n-\varepsilon_{T}(G)$. Conversely, Let $D$ be connected dominating set. Since $\left\langle\mathrm{D}>\right.$ is connected. $\langle\mathrm{D}\rangle$ has a spanning tree $\mathrm{T}_{\mathrm{s}}$. A spanning tree $\mathrm{T}$ of $\mathrm{G}$ is formed by adding the remaining $n-\gamma_{c}(G)$ vertices of $V-D$ to $T_{s}$ and adding edges of $G$ such that vertex in $V$-D is adjacent to exactly one vertex in D. Now $T$ has at least $n-\gamma_{c}(G)$ pendant vertices. Thus $\varepsilon_{T}(G) \geq n-\gamma_{c}(G)$ or $\gamma_{c}(G) \geq n-\varepsilon_{T}(G)$. Hence $\gamma_{c}(G)=n-\varepsilon_{T}(G)$ and since $\varepsilon_{T}(G) \geq 2$ ie., $\gamma_{c}(G) \leq n-2$ 
Theorem 3.5: Let $G$ be connected fuzzy graph and have $n$ vertices $m$ edges if $n \geq 4, m \geq n$ and $G$ is not a circuit then $\gamma_{c}(G) \leq n-3$

Proof: If $\mathrm{G}$ is not a circuit then $\mathrm{T}$ is a spanning fuzzy tree of $\mathrm{G} . \mathrm{G}$ must have at least one vertex $V$ with degree at least 3. By theorem3.2, $\gamma_{c}(\mathrm{G}) \leq \mathrm{n}-3$

Theorem 3.6: For any connected fuzzy graph $\mathrm{G}, \gamma_{c}(\mathrm{G}) \leq \mathrm{n}-\Delta(\mathrm{G})$.

Proof : Let $\mathrm{u}$ be node of $\mathrm{G}$ such that the strong neighborhood of $\mathrm{u}$ is $\mathrm{N}_{\mathrm{s}}(\mathrm{u})=\{\mathrm{v} \in \mathrm{V}:(\mathrm{u}, \mathrm{v})$ is strong arc $\}$ equal to maximum cardinality of a strong neighborhood $\Delta_{\mathrm{s}}(\mathrm{G})$ then $\mathrm{V}-\mathrm{N}_{\mathrm{s}}(\mathrm{u})$ is a dominating set. Therefore

$\gamma(\mathrm{G}) \leq\left|\mathrm{V}-\mathrm{N}_{\mathrm{s}}(\mathrm{u})\right| \leq \mathrm{n}-\Delta_{\mathrm{s}}(\mathrm{G})$. The maximum cardinality of a strong neighborhood is maximum degree of $\mathrm{u}$ and $\gamma$ $(\mathrm{G}) \leq \gamma_{\mathrm{c}}(\mathrm{G})$ that is $\gamma_{\mathrm{c}}(\mathrm{G}) \leq \mathrm{n}-\Delta(\mathrm{G})$.

Corollary 3.7: For any fuzzy tree $\mathrm{T}, \gamma_{\mathrm{c}}(\mathrm{T})=\mathrm{n}-\Delta(\mathrm{T})$ then $\mathrm{T}$ has atmost one vertex of degree three or more.

Theorem 3.8 If $H$ is connected fuzzy spanning subgraph of $G$ then $\gamma_{c}(G) \leq \gamma_{c}(H)$

Proof: If $\mathrm{H}$ is connected spanning subgraph of a connected fuzzy graph $\mathrm{G}$ then every connected dominating set of $\mathrm{H}$ is also connected dominating set of $\mathrm{G}$ and $\mathrm{V}=\mathrm{V}_{1}$. The spanning subgraph of $\mathrm{G}$ has connected dominating set but need not be a minimum fuzzy dominating set. ie, $\gamma_{c}(G) \leq \gamma_{c}(H)$

\section{Connected Domination Number Of Square Fuzzy Graph}

In this section, we introduce a new notion called connected domination number of fuzzy square graph

Definition 4.1 The square fuzzy graph $G$ is $G^{2}=\left(V, E\left(G^{2}\right)\right)$. A set $D$ of $G^{2}$ is said to be connected dominating set of $\mathrm{G}^{2}$ if every vertex not in $\mathrm{D}$ is adjacent to at least one vertex in $\mathrm{D}$ and the sub graph $\mathrm{H}=\langle\mathrm{D}\rangle$ is connected. The minimum cardinality of a connected dominating set of $\mathrm{G}^{2}$ is called the connected domination number of fuzzy square graph $\mathrm{G}^{2}$ and denoted by $\gamma_{c}\left(\mathrm{G}^{2}\right)$. The fuzzy square graph $\mathrm{G}$ denoted by $\mathrm{G}^{2}$ has the same vertices as in $G$ and the vertices $u$ and $v$ are joined in $G^{2}$ if and only if they are joined in $G$ by a path of length is less than or equal to two.

Theorem 4.2: For any connected fuzzy graph $\mathrm{G}, \gamma_{\mathrm{c}}\left(\mathrm{G}^{2}\right) \leq \gamma_{\mathrm{c}}(\mathrm{G})$

Proof: For any connected fuzzy graph $\mathrm{G}$ with $\mathrm{n} \geq 3$. Let $\mathrm{P}$ be a path such that $\mathrm{P}:\left(\mathrm{u}_{0}, \mathrm{u}_{1}\right) \cdot\left(\mathrm{u}_{1}, \mathrm{u}_{2}\right) \ldots\left(\mathrm{u}_{\mathrm{n}-1}, \mathrm{u}_{\mathrm{n}}\right)$ where $\mu\left(u_{i-1}, u_{i}\right)>0 \forall$ i. Suppose $S=\left\{u_{i} \in V, \sigma\left(u_{i}\right)>0 \forall i\right\}$ be a connected dominating set of $G$ such that $S$

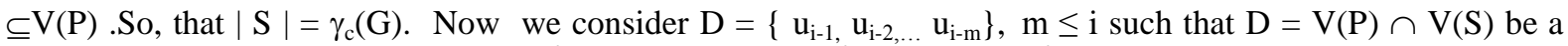
minimal connected dominating set of $G^{2}$. Hence $|D|=\gamma_{c}\left(G^{2}\right)$ clearly $\gamma_{c}\left(G^{2}\right) \leq \gamma_{c}(G)$

Theorem 4.3 : If $G$ is connected fuzzy graph $G$ then $\gamma_{c}\left(G^{2}\right) \leq 5 \gamma\left(G^{2}\right)-4$

Proof: Let $G$ be a connected fuzzy graph $G$ and Let $D$ be a dominating set of $G^{2}$ then the induced fuzzy subgraph $\langle D\rangle$ has at most $|D|$ components. Since $D$ is a dominating set of $G^{2}$. We can connect two of these components to one components by adding atmost four vertices to D. Hence we can construct a connected dominating set $\mathrm{S}$ of $\mathrm{G}^{2}$ such that $\mathrm{D} \subseteq \mathrm{S}$ in atmost $|\mathrm{D}|-1$ steps by adding atmost four times of $|\mathrm{D}|-1$ vertices to D. Consequently $\gamma_{c}\left(G^{2}\right) \leq|S| \leq|D|+4(|D|-1)=5|D|-4$ and if we choose $D$ such that $|D|=\gamma\left(G^{2}\right)$

Theorem 4.4 : For any connected fuzzy graph $\mathrm{G}:(\sigma, \mu), \gamma_{c}(G)-\gamma(G) \leq n-\gamma_{c}\left(G^{2}\right)$

Proof: Let $H=\left\{v_{n} \in V, \sigma\left(v_{n}\right)>0\right\}$ be a minimal dominating set of $G$. Suppose the subgraph $\langle H\rangle$ is connected then $\mathrm{H}$ is connected dominating of $\mathrm{G}$.Further if the subgraph $\langle\mathrm{H}\rangle$ is disconnected then there exist another vertex set $\mathrm{J}=\left\{\mathrm{v}_{\mathrm{i}} \in \mathrm{V}, \sigma\left(\mathrm{v}_{\mathrm{i}}\right)>0\right\}$ where $\mathrm{J} \subseteq \mathrm{V}(\mathrm{G})-\mathrm{H}$ whose vertices are at distance one to the vertices in $\mathrm{H}$ such that the subgraph $\langle\mathrm{HU} \mathrm{J}\rangle$ is connected. Clearly $\mathrm{H} \mathrm{U} \mathrm{J}$ is a connected dominating set of $\mathrm{G}$. Since $\mathrm{V}(\mathrm{G})=\mathrm{V}\left(\mathrm{G}^{2}\right)$ there exists a vertex set $\mathrm{D}=\left\{\mathrm{v}_{\mathrm{k}} \in \mathrm{V}\right\} \subseteq \mathrm{H}$ U J. Clearly it follows that $|\mathrm{HU} \mathrm{J}|-|\mathrm{H}| \leq \mathrm{n}-|\mathrm{D}|$ there $\gamma_{c}(G)-\gamma(G) \leq n-\gamma_{c}\left(G^{2}\right)$.

Theorem 4.5 : Let $\mathrm{G}$ be connected fuzzy graph and $\mathrm{H}$ be any connected spanning fuzzy subgraph of $\mathrm{G}$ then every connected dominating set of $\mathrm{H}^{2}$ is also a connected dominating set of $\mathrm{G}^{2}$ and Hence $\gamma_{\mathrm{c}}\left(\mathrm{G}^{2}\right) \leq \gamma_{\mathrm{c}}\left(\mathrm{H}^{2}\right)$ Proof : Suppose $\mathrm{H}$ is totally disconnected or disconnected with at least one component as an isolated vertex. Then we consider $\mathrm{V}(\mathrm{G})=\left\{\mathrm{v}_{\mathrm{n}}, \sigma\left(\mathrm{v}_{\mathrm{n}}\right)>0, \mathrm{n}=1,2, \ldots\right\}$ and $\mathrm{S}=\left\{\mathrm{V}_{\mathrm{i}}\right\}, 1 \leq \mathrm{i} \leq \mathrm{n}$ such that $\mathrm{S} \subset \mathrm{V}(\mathrm{G})$ in such a way that every vertex of $\mathrm{V}-\mathrm{S}$ are at a distance of at most two with respect to the corresponding vertices of $\mathrm{S}$ in $\mathrm{G}$ which gives a connected minimal connected dominating set in $G^{2}$. Let $v_{i} v_{j}$ be any edge of $G$ such that $i<j$ and for all $i, j=1,2, . .(n-1)$. Suppose $H$ is minimal connected spanning subgraph of $G$ and $E(H)=E(G)-v_{i} v_{j}$. Since 
$\mathrm{V}\left(\mathrm{G}^{2}\right)=\mathrm{V}\left(\mathrm{H}^{2}\right)$ then $\mathrm{S}$ is also a minimal connected dominating set of both $\mathrm{G}^{2}$ and $\mathrm{H}^{2}$ which gives the equality $\gamma_{c}\left(G^{2}\right)=\gamma_{c}\left(H^{2}\right)$. If $H$ is totally disconnected then $V\left(G^{2}\right)=V\left(H^{2}\right)$ such that $E\left(H^{2}\right)=\Phi$,and $\gamma_{c}\left(G^{2}\right)=\gamma_{c}\left(H^{2}\right)=n$, where $n$ is the number of vertices in $G$. Now one can easily verify that $\gamma_{c}\left(G^{2}\right) \leq \gamma_{c}\left(H^{2}\right)$

\section{Conclusion}

The connected dominating number of fuzzy square graph is defined. Theorems related to this concept are derived and the relation between connected dominating number of fuzzy graphs and connected dominating number of fuzzy square graphs are established.

\section{References}

[1] Bhattacharya, Some remarks on fuzzy graphs, Pattern Recognition letter 6(1987), 297-302.

[2] J.N.Moderson and P.S.Nair, Fuzzy graphs and Fuzzy Hyper graphs, Physica verlag(2000).

[3] T.W.Haynes,S.T.Hedetniemi and P.J.Slater (Eds), Domination in graphs Advanced Topics,Marcel Dekkar inc,Newyork 1998.

[4] A.Rosenfeld, L.A.Zedeh.K.S.Fu.K.Tanaka.,M.Shimura(Eds), Fuzzy sets and their Application to cogenitive and Decision processes Academic press, Newyork (1975),77-95.

[5] F.Harary(1972), Graph theory,Adisen-wesley Reading mass.

[6] A.Somasundaram and S.Somasundaram,Domination in Fuzzy graph-I.,Pattern Recognition letter 19(9) (1998) ,77-95.

[7] M.H.Muddebihal,G.Srinivasa, Bounds connected domination in squares of graph,Inter.Jour.sci. and Tech.vol 1.No.4(2012),170-175

[8] Laura A.sanchis,On the number of edges in graph with a given connected domination number, Elsevier,Discrete Mathematics 214(2000), 193-210.

[9] D.Antony Xavior, D.Florence Isido, V.M.Chitra, On Domination in Fuzzy graph .Inter.journal of com. Algorithm vol 02(2013), 248-250.

[10] C.Y.Ponnappan, S.Basheer Ahamed.P.Surulinathan, The stong non split domination number of fuzzy graphs Inter.journal of com. and oraganisation Trends, vol8(2014),66-69.

[11] K.R.Bhutani, Strong arc in Fuzzy graphs,Information science,152(1989),319-322.

[12] A.Nagor Gani and V.T.chandrasekaran, Domination in Fuzzy graph, Advances in Fuzzy sets and system1 (1)(2006), 17-26.

[13] T.W.Haynes,S.T.Headetniemi, P.J. Slater, Fundanmental Domination in Graph,Marcel Dekkeel Newyork 1998

[14] F.Harary and I.C.Ross(1960) The square of a tree, Bell system Tech,J.,39,641-647. 